\title{
Differences in the Gas and Dust Distribution in the Transitional Disk of a Sun-like Young Star, PDS 70
}

\author{
Zachary C. Long $^{1}$ (D), Eiji Akiyama ${ }^{2}$ (D) , Michael Sitko ${ }^{1,3}$ (D), Rachel B. Fernandes ${ }^{4}$, Korash Assani ${ }^{1}$, Carol A. Grady ${ }^{5,6}$, Michel Cure ${ }^{7}$, \\ William C. Danchi ${ }^{6}$, Ruobing Dong ${ }^{8,26}$ (D), Misato Fukagawa ${ }^{9}$ (D), Yasuhiro Hasegawa ${ }^{10}$, Jun Hashimoto ${ }^{2}$, Thomas Henning ${ }^{11}$, \\ Shu-Ichiro Inutsuka ${ }^{12}$ (D), Stefan Kraus ${ }^{13}$ (D), Jungmi Kwon ${ }^{14}$ (D), Carey M. Lisse ${ }^{15}$ (D), Hauyu Baobabu Liu ${ }^{16}$, Satoshi Mayama ${ }^{17}$, \\ Takayuki Muto $^{18}$, Takao Nakagawa ${ }^{19}$, Michihiro Takami ${ }^{20}$ (D), Motohide Tamura ${ }^{2,21,22}$ (D), Thayne Currie ${ }^{23}$ (D), \\ John P. Wisniewski ${ }^{24}$, and Yi Yang ${ }^{2,25}$ \\ ${ }^{1}$ Department of Physics, University of Cincinnati, Cincinnati, OH 45221, USA \\ ${ }^{2}$ National Astronomical Observatory of Japan, 2-21-1, Osawa, Mitaka, Tokyo, 181-8588, Japan \\ ${ }^{3}$ Center for Extrasolar Planetary Studies, Space Science Institute, 4750 Walnut Street, Suite 205, Boulder, CO 80301, USA \\ ${ }^{4}$ Lunar and Planetary Laboratory, University of Arizona, Tucson, AZ 85719, USA \\ ${ }^{5}$ Eureka Scientific, 2452 Delmer Street, Suite 100, Oakland CA 96402, USA \\ ${ }^{6}$ Exoplanets and Stellar Astrophysics Laboratory, NASA Goddard Space Flight Center, Greenbelt, MD, USA \\ ${ }^{7}$ Instituto de Física y Astronomía, Universidad de Valparaíso, Chile \\ ${ }^{8}$ Steward Observatory, University of Arizona, Tucson, AZ, 85719, USA \\ ${ }^{9}$ Division of Particle and Astrophysical Science, Graduate School of Science, Nagoya University, Furo-cho, Chikusa-ku, Nagoya, Aich 464-8602, Japan \\ ${ }_{10}$ Jet Propulsion Laboratory, California Institute of Technology, Pasadena, CA 91109, USA \\ ${ }^{11}$ Max Planck Institute for Astronomy, Königstuhl 17, D-69117 Heidelberg, Germany \\ ${ }_{12}^{12}$ Department of Physics, Graduate School of Science, Nagoya University, Nagoya 464-8602, Japan \\ ${ }^{13}$ School of Physics, Astrophysics Group, University of Exeter, Stocker Road, Exeter EX4 4QL, UK \\ ${ }^{14}$ Institute of Space and Astronautical Science, Japan Aerospace Exploration Agency, 3-1-1 Yoshinodai, Chuo-ku, Sagamihara, Kanagawa 252-5210, Japan \\ ${ }^{15}$ Space Exploration Sector, JHU-APL, 11100 Johns Hopkins Road, Laurel, MD 20723, USA \\ ${ }^{16}$ European Southern Observatory (ESO), Karl-Schwarzschild-Straße 2, D-85748 Garching, Germany \\ ${ }^{17}$ The Center for the Promotion of Integrated Sciences, The Graduate University for Advanced Studies (SOKENDAI), Shonan International Village, Hayama-cho, \\ Miura-gun, Kanagawa 240-0193, Japan \\ ${ }^{18}$ Division of Liberal Arts, Kogakuin University, 1-24-2 Nishi-Shinjuku, Shinjuku-ku, Tokyo, 163-8677, Japan \\ ${ }^{19}$ Institute of Space and Astronautical Science, Japan Aerospace Exploration Agency, Kanagawa 252-5210, Japan; nakagawa@ ir.isas.jaxa.jp \\ ${ }^{20}$ Institute of Astronomy and Astrophysics, Academia Sinica, P.O. Box 23-141, Taipei 10617, Taiwan \\ ${ }^{21}$ Department of Astronomy and RESCUE, The University of Tokyo, 7-3-1, Hongo, Bunkyo-ku, Tokyo, 113-0033, Japan \\ ${ }^{22}$ Astrobiology Center of NINS, 2-21-1, Osawa, Mitaka, Tokyo, 181-8588, Japan \\ ${ }^{23}$ National Astronomical Observatory of Japan, Subaru Telescope, National Institutes of Natural Sciences, Hilo, HI 96720, USA \\ ${ }^{24}$ Homer L. Dodge Department of Physics, University of Oklahoma, Norman, OK 73071, USA \\ ${ }^{25}$ Department of Astronomical Science, The Graduate University for Advanced Studies (SOKENDAI), 2-21-1 Osawa, Mitaka, Tokyo 181-8588, Japan \\ Received 2017 November 13; revised 2018 March 23; accepted 2018 March 26; published 2018 May 14
}

\begin{abstract}
We present ALMA $0.87 \mathrm{~mm}$ continuum, $\mathrm{HCO}^{+} J=4-3$ emission line, and $\mathrm{CO} J=3-2$ emission line data of the disk of material around the young, Sun-like star PDS 70. These data reveal the existence of a possible twocomponent transitional disk system with a radial dust gap of 0 ". $42 \pm 0$." 05 , an azimuthal gap in the $\mathrm{HCO}^{+}$ $J=4-3$ moment zero map, as well as two bridge-like features in the gas data. Interestingly these features in the gas disk have no analog in the dust disk making them of particular interest. We modeled the dust disk using the Monte Carlo radiative transfer code HOCHUNK3D using a two-disk component. We find that there is a radial gap that extends from 15 to 60 au in all grain sizes, which differs from previous work.
\end{abstract}

Key words: astrochemistry - planetary systems - protoplanetary disks - stars: low-mass - stars: pre-main sequence

\section{Introduction}

Large interferometer telescopes such as the Atacama Large Millimeter/submillimeter Array (ALMA) and the Very Large Telescope Interferometer (VLTI) have recently facilitated an increasingly detailed examination of circumstellar disks around young stars. They have the ability to detect molecular emission spectra, as well as dust continuum emission, at very high angular resolutions even at the millimeter and submillimeter wavelengths. The much higher angular resolution, when compared to a single telescope at similar wavelengths, allows us to probe closer to the central star, and study the spatial relationships between gas and dust on a finer scale than ever before.

PDS 70 is a pre-main-sequence star (PMS) with spectral type K5 assumed to be part of the Centaurus association and

\footnotetext{
${ }^{26}$ Bok Fellow.
}

estimated to be at a distance of $140 \mathrm{pc}$ (Riaud et al. 2006). It has previously been imaged in both $H$-band polarized light using Subaru (Hashimoto et al. 2012), as well as more recently at $1.3 \mathrm{~mm}$ with the Submillimeter Array (SMA; Hashimoto et al. 2015). In this paper we present a detailed examination of the circumstellar disk of PDS 70 in the $0.87 \mathrm{~mm}$ continuum, $\mathrm{CO} J=3-2$ emission, and $\mathrm{HCO}^{+} J=4-3$ emission based on ALMA observations. These data are at a much higher resolution than the SMA data, 0." $19 \times 0$ ". 15 compared to 0 . $88 \times 0$." 43 , and provide us with a good probe of the larger grains within the disk. They also allow us to examine the gas distribution and detect fine structures within disk gas. This is of particular interest to our study due to the existence of features that appear in the gas disk but are not present in the dust disk. PDS 70 has proven to be a system with a different structure in each of our continuum, $\mathrm{CO} J=3-2, \mathrm{HCO}^{+}$ $J=4-3$ measurements presented below. In addition to our 


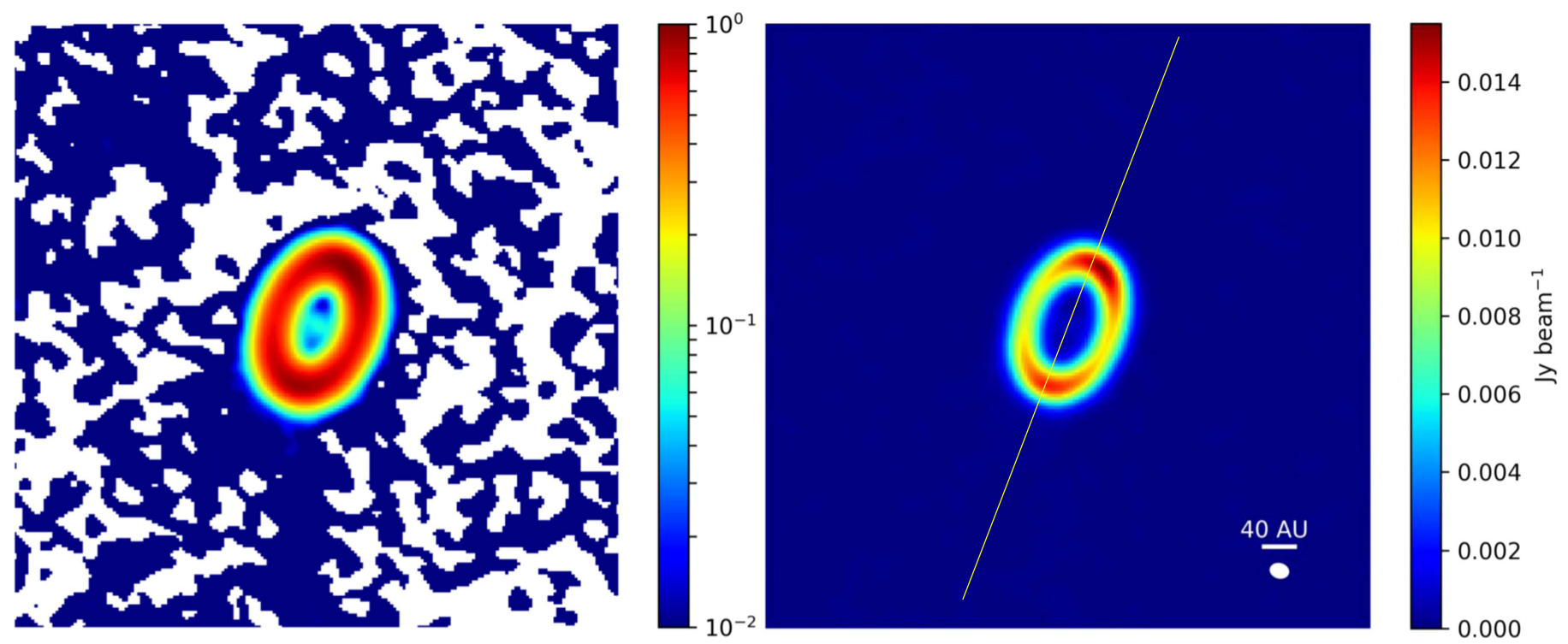

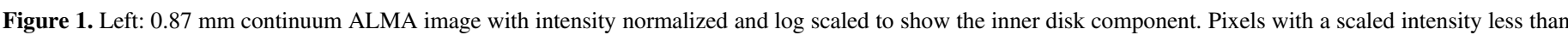

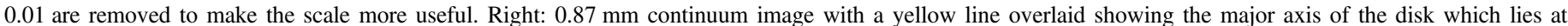
$159^{\circ} \pm 5^{\circ}$. North is up and east is left in these images.

observational data we present a Monte Carlo radiative transfer (MCRT) model of the disk using HOCHUNK3D (Whitney et al. 2013) to simultaneously fit existing imagery as well as the spectral energy distribution (SED) of PDS 70. Following the discussion of the data and model, we also present additional SpeX and REM observations of PDS 70 as well as an analysis of these data sets in the Appendix including gas accretion, mass, and age estimates.

\section{Observations and Data Reduction}

Observations were conducted on 2016 August 14 and 18 in the ALMA cycle 3 program (No. 2015.1.000888.S). We used 38 to 39 of the $12 \mathrm{~m}$ diameter antennas in the observation. The array configuration provides a maximum and minimum baseline length of $1.5 \mathrm{~km}$ and $15.1 \mathrm{~m}$, respectively. Sky conditions were relatively stable with the precipitable water vapor between $0.45 \mathrm{~mm}$ and $0.82 \mathrm{~mm}$. The system temperature was between $90 \mathrm{~K}$ and $280 \mathrm{~K}$ during observations. The correlator was tuned at the center frequency of $345.761 \mathrm{GHz}$ for $\mathrm{CO} J=3-2,356.698 \mathrm{GHz}$ for $\mathrm{HCO}^{+} J=4-3$, and $343.955 \mathrm{GHz}$ and $355.497 \mathrm{GHz}$ for continuum observations in the dual polarization mode. The effective bandwidths for both emission lines are $468.75 \mathrm{MHz}$ and their frequency resolution is $244.141 \mathrm{kHz}$, corresponding to approximately $0.21 \mathrm{~km} \mathrm{~s}^{-1}$ in the velocity resolution.

Data reduction was performed using version 4.7 of the Common Astronomy Software Applications package (CASA). A quasar J1427-4206 was used for bandpass, flux, and phase calibrations, and another quasar, J1407-4302, was selected as a second calibrator. We used the task CLEAN in CASA and a continuum image was generated by combining the line-free channels in all of the spectral windows. The self-calibration was performed by applying the obtained continuum image as a model, and gain calibration was repeated until the rms reached minimum. The gain table obtained after the self-calibration of the continuum data was applied to both the $\mathrm{CO} J=3-2$ and $\mathrm{HCO}^{+} J=4-3$ to generate self-calibrated visibilities. Because the line and continuum observations were simultaneously conducted, and the atmospheric variation equally affects the phase variation of line and continuum data, the calibration table obtained from the continuum data can be applied to line emission calibration. Natural weighting was applied in both continuum and line imaging for better sensitivity. The achieved rms was $0.0551 \mathrm{mJy} \mathrm{beam}^{-1}$ for the continuum, $1.204 \mathrm{mJy}$ beam $^{-1}$ for CO $J=3-2$, and $1.118 \mathrm{mJy}_{\text {beam }}{ }^{-1}$ for $\mathrm{HCO}^{+}$ $J=4-3$. The field of view was approximately $18^{\prime \prime}$ and the final synthesized beam of approximately 0 " $19 \times 0$ " 15 for the continuum image and CO $J=3-2$ map, and 0 ". $18 \times 0$ ". 15 for the $\mathrm{HCO}^{+} J=4-3$ map after calibration and flagging as denoted in Figures 1 and 2. The total integration time on the source after flagging is about 56 minutes.

\section{Results}

\section{1. $0.87 \mathrm{~mm}$ Continuum Measurements}

The $0.87 \mathrm{~mm}$ continuum image gives us a good measure of the radial extent of the dust disk, the flux density, and reveals some other interesting features. The major axis of the disk lies at $\sim 159^{\circ}$ east of north, measured via ellipse fitting of the brightest emission along the ring of the disk (Figure 1), and measures a radial distance of 0 ". $42 \pm 0$ ". 05 ( $\sim 60 \mathrm{au}$ ) from the center at the edge and $0 . / 79 \pm 0.05(\sim 110 \mathrm{au})$ at the outer edge. Both edges were found by measuring a distance equal to half the width of the beam size from the inner and outer edges of the disk that are detectable above noise. The measured flux density is $233 \pm 3 \mathrm{mJy}$ integrated at a radius of 1 ." 00 , past which there is no detectable emission above $\sim 2 \sigma$. In Figure 1 the disk brightness is clearly not symmetrical about the minor axis, similar to what is seen in Kraus et al. (2017), with the northwest side $\sim 15 \%$ brighter than the southeast side.

There also appears to be material visible near the center of the disk in the continuum, seen more clearly in the log scaled image (Figure 1, left), which is separated from the outer disk by a radial gap. It is slightly larger than the resolution of the measurement, extending out approximately 0 !" 2 where the resolution of the instrument is 0 ". $19 \times 0$ " 15 and is likely an inner disk component. 

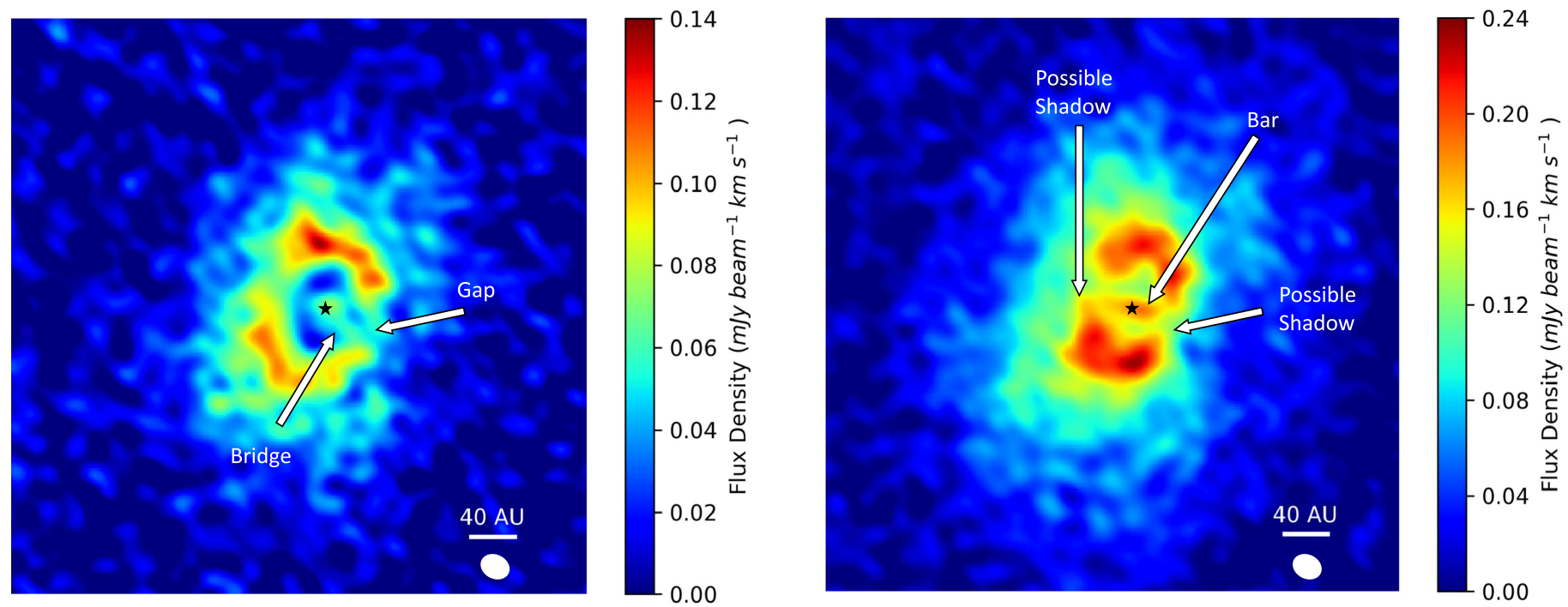

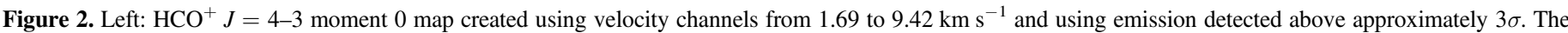

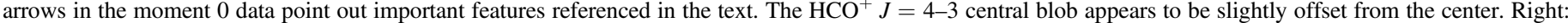

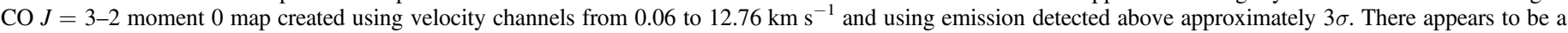

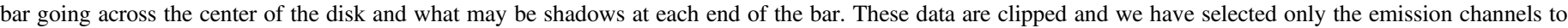
generate these maps. The black star is at the location of PDS 70 .

\subsection{1. $\mathrm{HCO}^{+} \mathrm{J}=4-3$ Measurements}

The $\mathrm{HCO}^{+} J=4-3$ moment 0 emission (Figure 2) reveals a similar gap to what is seen in the dust continuum (Figure 1), though it extends to only $\sim 33$ au while the outer edge of the $\mathrm{HCO}^{+} J=4-3$ moment 0 emission extends to $\sim 160 \mathrm{au}$. The gas disk also extends out farther than the dust though not as far as in many very young PMS stars such as DM Tau (Öberg et al. 2010). It contains a bright blob $(\sim 10 \sigma)$ to the north, as well as a bridge structure connecting the inner region and the outer disk in the southwest, and a gap due west, which lies directly adjacent to the bridge. There is also evidence of an azimuthal gap or depleted region to the northeast.

The bridge structure is likely to be real, with a signal-tonoise ratio of $\sim 5$. In order to help verify this we generated a rough model of the bridge and azimuthal gap (Figure 3 ). This was produced using the CASA 5.2 task simobserve with the thermal noise set to default and the antennas set to the ALMA cycle 4 configuration, alma.cycle4.3.cfg. The seed image for the model is a simple, two-component system generated using HOCHUNK3D with the bridge and depleted region added to the seed using image editing software. We found that in order to produce any asymmetric features to the degree of those seen in Figure 2, the inclusion of a dimmer region to reproduce the gap and a bridge connecting the inner and outer disks were needed.

The bridge feature itself appears to be mass transfer between the inner and outer disks similar to what is suggested for HD 142527 (Casassus et al. 2013). If this were the case we would expect to see a radial velocity component roughly perpendicular to the Keplerian motion of the disk which is not seen here (Figure 4). It has also been suggested that bridges such as this can be caused by a polar outflow of gas (Alves et al. 2017). Again this is likely not the case, however as there appears to be no significant change in velocity in the region of the bridge, and it truncates at the outer disk instead of continuing to expel matter outside of the disk. Its close proximity to the western gap suggests that the two are related and could be due to large body formation which has been shown to cause both depleted regions as well as spiral arms in other disks (Edgar \& Quillen 2008; Dong et al. 2015; Matter et al. 2016). Interestingly enough however, there is no evidence of the bridge feature or an azimuthal gap in the $0.87 \mathrm{~mm}$ continuum ring or in $H$-band polarized imagery (Dong et al. 2012).

As can be seen in Figure 2, the inner $\mathrm{HCO}^{+} J=4-3$ disk appears slightly offset from the inner dust disk. To verify this we created radial traces of the continuum and moment 0 gas data, shown in Figure 5. There also may be some asymmetry in the outer gas disk as the $\mathrm{HCO}^{+} J=4-3$ moment 0 peak intensities lie at different distances from the center of the disk. In the $\mathrm{HCO}^{+} J=4-3$ moment 1 and 2 maps, the central region was clipped out due to excess noise in the region (Figure 6).

\subsection{2. $\mathrm{CO} J=3-2$ Measurements}

The CO $J=3-2$ moment 0 emission lies interior to the continuum and $\mathrm{HCO}^{+} J=4-3$ emission, exhibiting little to no gap between the inner and outer disk. Similar to the $\mathrm{HCO}^{+}$ $J=4-3$ moment 0 data, this disk extends further out than the dust disk, to $\sim 210 \mathrm{au}$, but again not as much as much younger PMS stars. This is promising for planet formation similar to what is seen in our solar system as there is still mass within $40 \mathrm{au}$ of the star. The emission contains a blob in the $\mathrm{S}$ similar to what is seen in $\mathrm{HCO}^{+} J=4-3$, but almost directly opposite its location in the disk.

Again, similar to what is seen in $\mathrm{HCO}^{+} J=4-3$, there is a bar feature toward the center of the image. This, however, appears to extend to either side of the disk and, as CO $J=3-2$ is generally considered optically thick in protoplanetary disks, could be causing the dimmed regions seen at either end of the bridge. It appears that the bridge feature lies within the plane of the disk as the moment 1 data shows no deviation from Keplerian rotation in the region of the bridge (Figure 4).

The CO moment 2 map contains a butterfly pattern surrounding the central region. This pattern exists in the same location as the four bright regions in the moment 0 data, but are due to crowded isovelocity curves in the moment 1 data and are likely not related. 

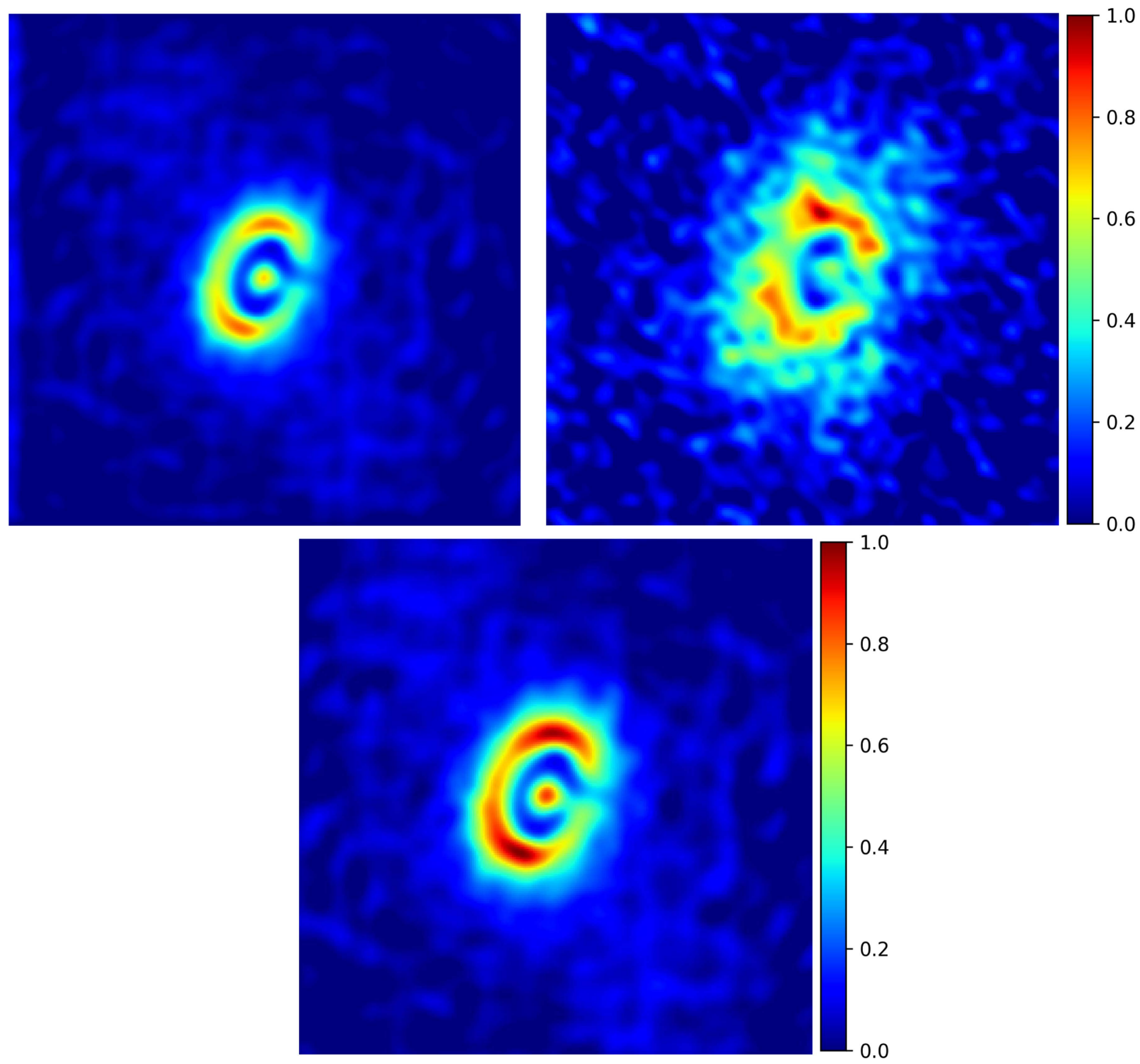

Figure 3. Left: simulated model image produced using the simobserve task in CASA 5.2. The image is displayed on the same scale as the right image with the peak brightness adjusted to match the brightness of the southern section of the right image. This was done to better show the relative brightness of the ring and central region as the bright blob in the north of the right image makes the comparison unclear. Right: $\mathrm{HCO}^{+} J=4-3$ moment 0 map normalized to the brightest pixel in the image. Bottom: the same image as the top left, but normalized to the brightest pixel in the image.

\subsection{Model}

\subsubsection{Initial Model}

For our modeling we used the MCRT code HOCHUNK3D (Whitney et al. 2013). This code allows for two distinct grain populations as well as an envelope which can be manipulated into a third distinct grain population if necessary. The locations of these populations can also be changed to produce radial gaps in the disk.

We used a model similar to those of Hashimoto et al. (2015) and Dong et al. (2012) as our starting point. For the star we used a PMS star with spectral type K5, a radius of $1.39 R_{\odot}$, and a temperature of $4400 \mathrm{~K}$, as suggested by Gregorio-Hetem \&
Hetem (2002). We also use a mass of $0.82 M_{\odot}$, estimated by Riaud et al. (2006) using spectral type and further supported by dynamical fitting (Hashimoto et al. 2015). We then constructed a disk consisting of two rings with a large gap in the middle, a mass of $4.5 M_{\mathrm{J}}$, an inclination of $50^{\circ}$ (Hashimoto et al. 2012), and a large grain to total a grain mass ratio of 0.9667 in order to decrease the optical thickness of the small grains and increase the 10 and $20 \mu \mathrm{m}$ silicate peaks. We also used the same grain types as the Hashimoto et al. (2015) model for the large and small grains that make up the disk.

Our initial model consisted of one that included both grain types in the inner disk that extended from the sublimation radius $\sim 0.05$ au to $1 \mathrm{au}$, which was used to reproduce the 

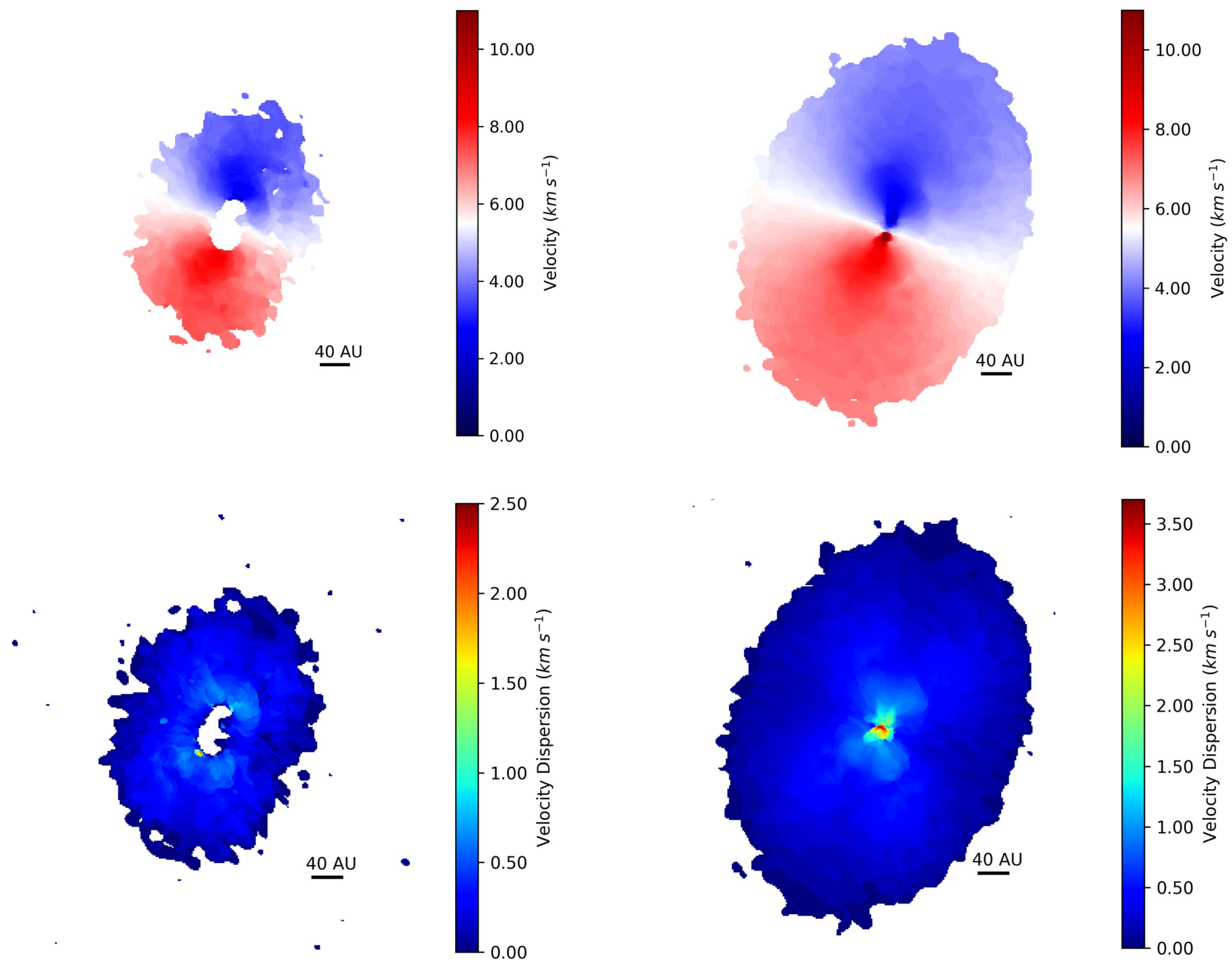

Figure 4. Left top: $\mathrm{HCO}^{+} J=4-3$ moment 1 map created using velocity channels from 1.69 to $9.42 \mathrm{~km} \mathrm{~s}^{-1}$ and emission above $0.028 \mathrm{Jy}$ beam ${ }^{-1}$. Left bottom: $\mathrm{HCO}^{+} J=4-3$ moment 2 map created using velocity channels from 1.69 to $9.42 \mathrm{~km} \mathrm{~s}^{-1}$ and emission above $0.022 \mathrm{Jy}$ beam ${ }^{-1}$. Right top: CO $J=3-2$ moment 1 map created using velocity channels from 0.06 to $12.76 \mathrm{~km} \mathrm{~s}^{-1}$ and emission above $0.023 \mathrm{Jy}$ beam ${ }^{-1}$. Right bottom: CO J=3-2 moment 2 map created using velocity channels from 0.06 to $12.76 \mathrm{~km} \mathrm{~s}^{-1}$ and emission above $0.02 \mathrm{Jy} \mathrm{beam}^{-1}$. All of the maps were created using CASA 5.1.

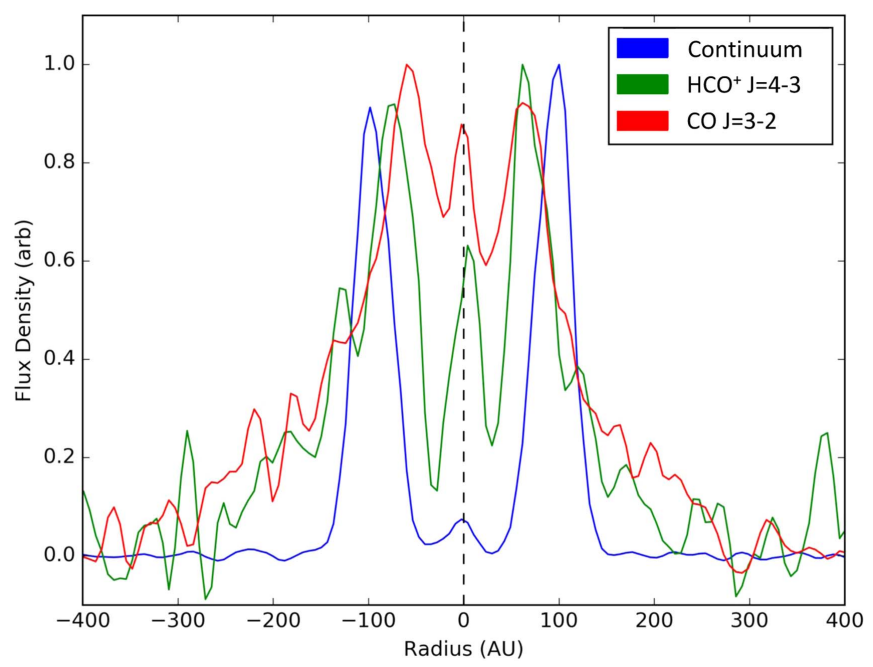

Figure 5. Surface brightness slices along the major axis of the $0.87 \mathrm{~mm}$ continuum image (blue), $\mathrm{HCO}^{+} J=4-3$ moment 0 data (green), and $\mathrm{CO}$ $J=3-2$ moment 0 data (red).
near-IR emission seen in the SED. This was followed by a gap that extended to $65 \mathrm{au}$ for the smaller grains and 80 au for the larger grains based on the $H$-band data (Dong et al. 2012), the $1.3 \mathrm{~mm}$ data (Hashimoto et al. 2015), and a distance of $140 \mathrm{pc}$ (Riaud et al. 2006). This reproduced a fit to both the SED and $1.3 \mathrm{~mm}$ image seen in Hashimoto et al. (2015) as well as Dong et al. (2012).

\subsubsection{Model Fitting}

Since the population of small grains is constrained by the character of the silicate emission bands near the 10 and $20 \mu \mathrm{m}$ wavelengths, we also include data obtained with the Spitzer InfraRed Spectrograph (IRS) in our analysis. Both of these features are the result of warm silicate emission close to the star and required modification of the initial model to provide a reasonable fit.

It is important to note also that the $A K A R I 18 \mu \mathrm{m}$ point disagrees with the Spitzer spectrum despite the 13.9-23.6 $\mu \mathrm{m}$ bandpass of the filter, and could indicate that either there is 

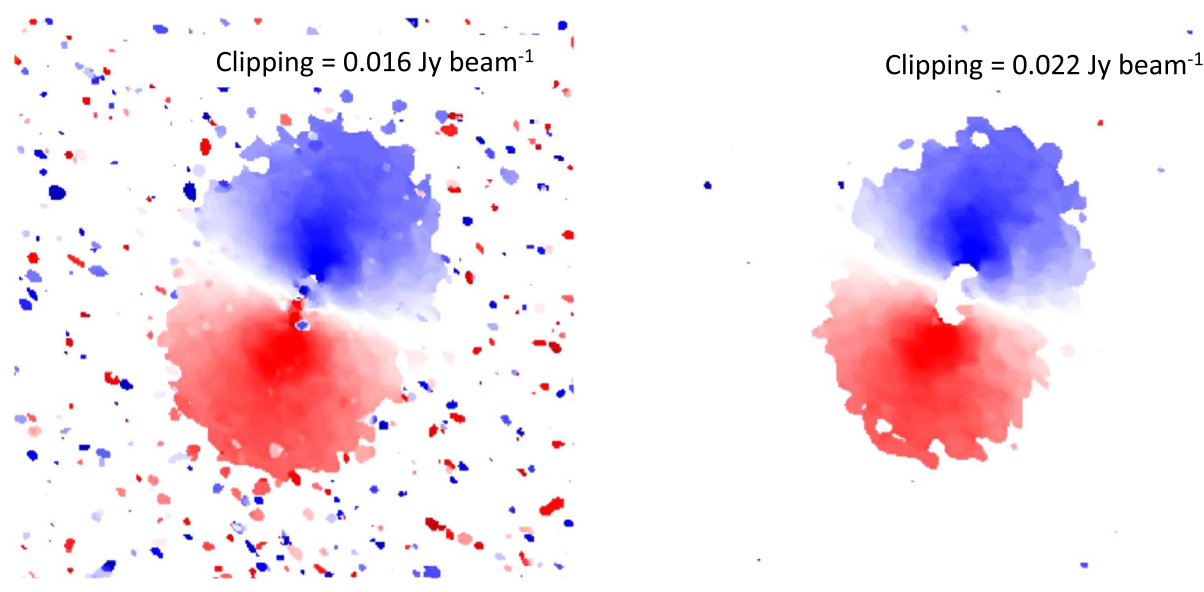

Clipping $=0.028 \mathrm{Jy} \mathrm{beam}^{-1}$

Figure 6. $\mathrm{HCO}^{+} J=4-3$ moment 1 maps at various clipping thresholds.

some variability of the disk or that one of the data sets is incorrect. The grains that produce this peak lie approximately $0.5-3$ au from the star which, assuming that the star is $0.82 M_{\odot}$, suggests orbital periods of $\sim 0.39-5.7$ years. It is possible therefore, that the disk could have changed between the two data sets. Additionally, it has been suggested that thermal instabilities due to active accretion could result in variability (Sitko et al. 2012). However PDS 70 seems to exhibit no accretion (Appendix).

A second difference is the inclusion of the newly acquired of ALMA $0.87 \mathrm{~mm}$ continuum data described in Section 2. The ALMA data have a much higher resolution than the SMA data used by Hashimoto et al. (2015), which allows for much better spatial estimates of the disk. These data exhibit a different, $\sim 60$ au gap radius and lack the highly asymmetric spatial distributions in the SMA dust emission. This is in agreement with the gap radius found in scattered light (Dong et al. 2012) which suggests that the apparent difference in gap radius for the two grain populations is not real.

To fit the model with these new constraints we included a less dense, though still optically thick, inner disk comprised of only small grains that extends from the sublimation radius to $15 \mathrm{au}$. We also raised the disk mass from $4.5 M_{\mathrm{J}}$ to $\sim 7.3 M_{\mathrm{J}}$ to fit the ALMA $0.87 \mathrm{~mm}$ point and raised the large grains to a total grain ratio of 0.995 . This produced very strong silicate peaks, but does not reach the depth of the dips before the 10 and $20 \mu \mathrm{m}$ peaks. The inner disk is followed by a gap extending to $60 \mathrm{au}$ and an outer disk extending from 60 au to $110 \mathrm{au}$, based on the $0.87 \mathrm{~mm}$ continuum image. The higher spatial resolution of the ALMA data also allows for better comparison of the disk inclination to the model. We found the apparent inclination of the disk is best reproduced with a model inclination of $45^{\circ}$ from face on.

In order to probe the optical depth of the outer disk we examined the thickness of the disk, defined by $z=C_{1} r^{b}$, where $\mathrm{z}$ is the scale height (thickness) of the disk, $C_{1}$ is a constant, $\mathrm{r}$ is the radial distance from the star, and $\mathrm{b}$ is the flaring exponent (Whitney et al. 2003). Similarly the radial density is defined by $\rho=C_{2} r^{-a}$, where $\rho$ is the density of the disk, $C_{2}$ is a constant, $\mathrm{r}$ is the radial distance from the star, and a is the density exponent (Whitney et al. 2003). To match the SED we find $b=1.15 \pm 0.02$ and $a=2.25 \pm 0.02$ for the large grains in our disk and $\mathrm{b}=1.15 \pm 0.02$ and $\mathrm{a}=1.5 \pm 0.02$ for the small grains. Based on these parameters we find that the optical depth of the outer disk

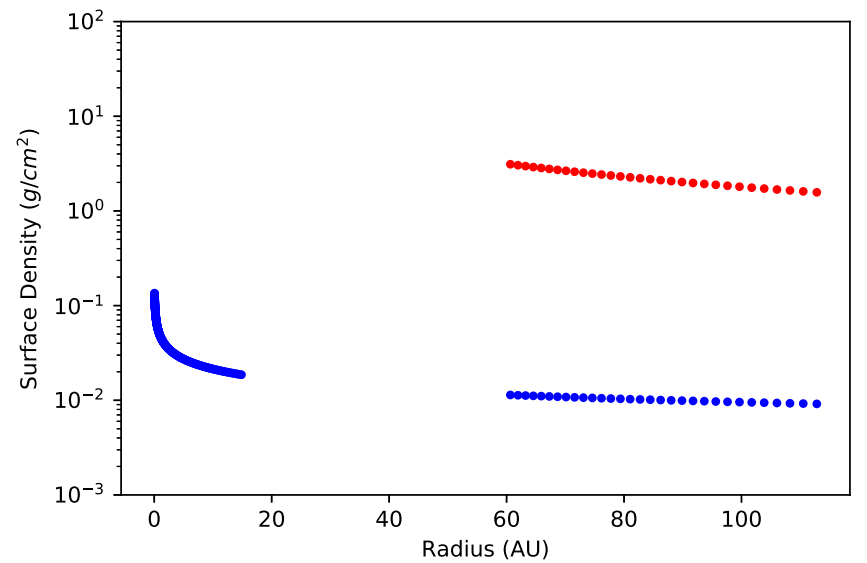

Figure 7. Surface density profile for the outer disk of PDS 70. The blue dotted line is for the small grain disk and the red dotted line is for the large grains.

is $\tau \sim 100 \mathrm{au}$, which is larger than the thickness of the outer disk suggesting that the disk is mostly optically thin at $0.87 \mathrm{~mm}$. These two exponents can also be used to calculate the surface density of the disk via the method described in Equation (15) $\left(\Sigma=\Sigma_{0} \varpi^{-p}\right)$ of Whitney et al. (2013) where $\Sigma_{0}$ is a constant set by the mass of the disk, $\varpi$ is the cylindrical radius, and $p=\alpha-\beta$. The surface density profile for the disk can be seen in Figure 7.

Because of the higher resolution of our data and the differences seen between this and the SMA data (Hashimoto et al. 2015), such as the outer disk extent, we used the CO $J=3-2$ moment 1 data to find the dynamical mass of PDS 70 . Here we assumed a disk inclination of $45^{\circ}$, based on our modeling, and similar to what is found in Hashimoto et al. (2015) we see that a mass of $0.6-0.8 M_{\odot}$ (Figure 8 ) produces a good fit to the data and is in agreement with the Riaud et al. (2006) estimation. This falls within the range of $0.5-1.1 M_{\odot}$ found from comparing the location of PDS 70 to isochrones (Appendix) derived in Tognelli et al. (2011) and seen in Figure 16.

\subsubsection{Final Model}

To test the accuracy of our model, we compared the model SED to existing photometry and spectrum data (Figure 9), and the model images to both the $0.87 \mathrm{~mm}$ continuum data (Figure 10) and the $\mathrm{H}$-band data (Hashimoto et al. 2012; 

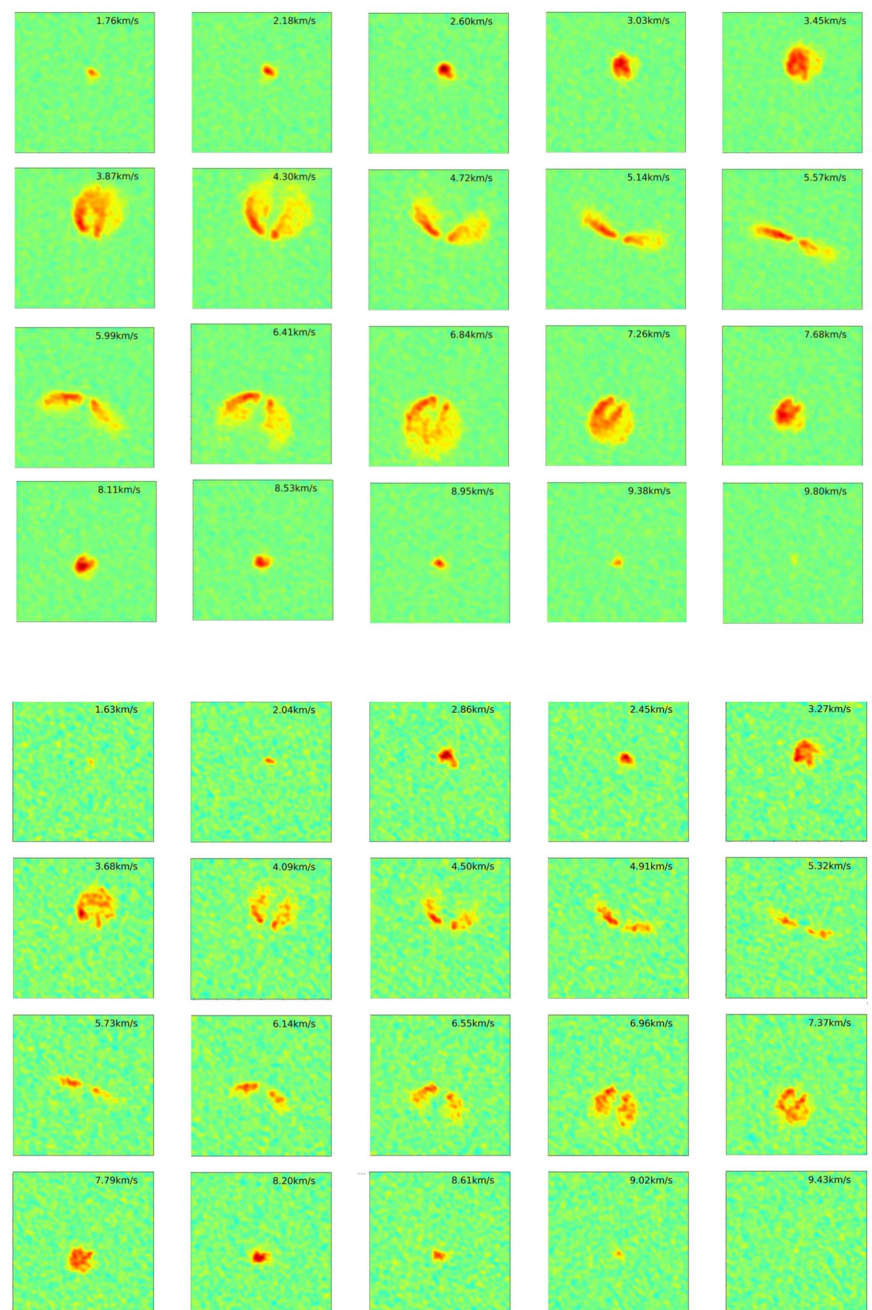
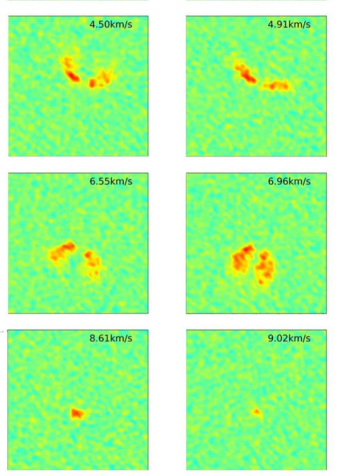
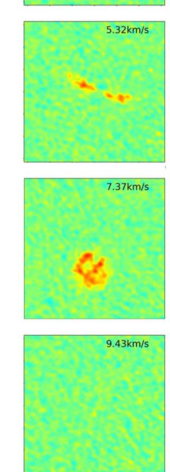

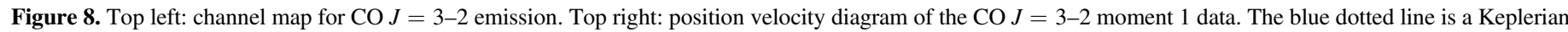

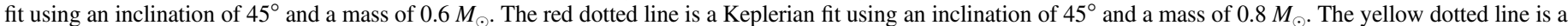

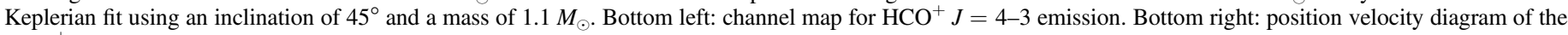
$\mathrm{HCO}^{+} J=4-3$ moment 1 map.

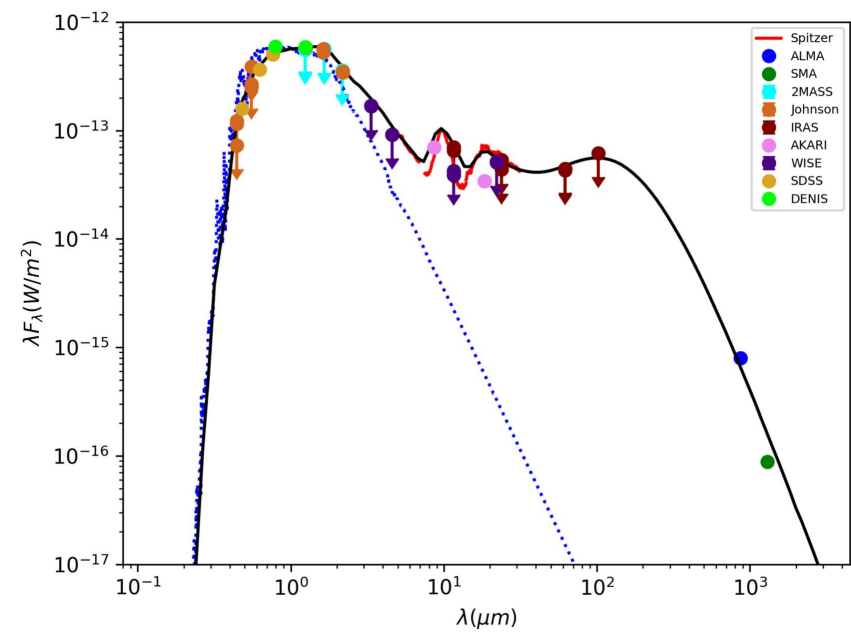

Figure 9. SED of PDS 70. The blue dotted line is the photosphere of a $4400 \mathrm{~K}$ star and the black line is the model fit.

Figure 11) after convolving with the beam shape and pointspread function of these data, respectively. In the case of the $0.87 \mathrm{~mm}$ model image we see a ratio of emission between the
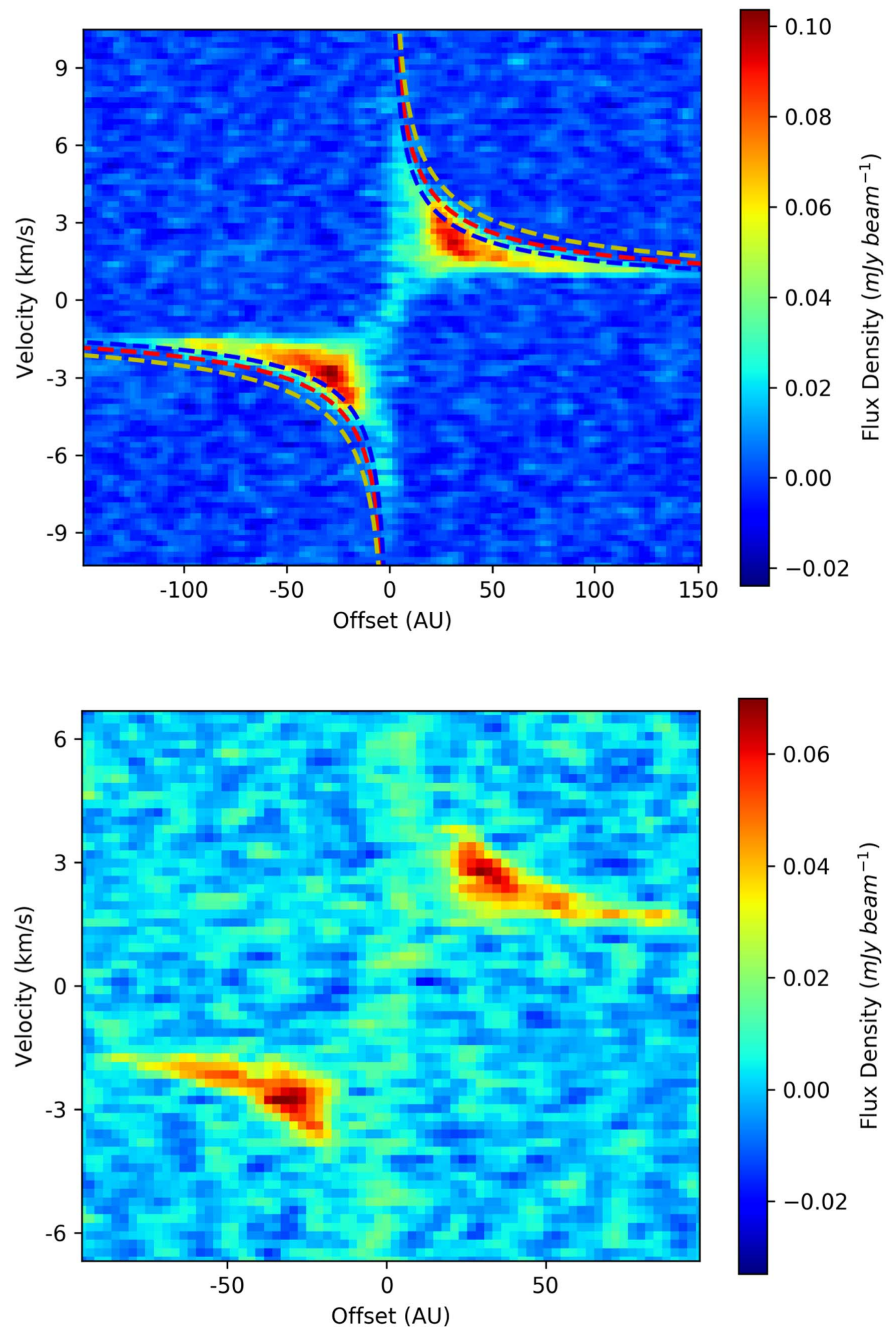

inner and outer disks that is in agreement with the ALMA image, $\frac{\text { Finner }}{\text { Fouter }} \sim 0.004$ in both cases, which suggests that the inner disk is essentially devoid of large grains. The $H$-band data reproduces the apparent dip in brightness seen in the northeast side of the disk (Figure 11). This dip is the result of co-planar shadowing from the the inner disk. The final model parameters can bee seen in Table 1.

\section{Discussion}

\subsection{Asymmetry in $0.87 \mathrm{~mm}$ Continuum Image}

If we assume that the $0.87 \mathrm{~mm}$ continuum is optically thin (Section 3.2.2), the difference in brightness should correspond to a difference in mass density between the two locations, suggesting that there is more mass density in the northwest side of the outer disk. There exists the possibility however, that the difference in brightness could be a result of apocenter glow (Pan et al. 2016), though we do not detect a pericenter offset in this system. Given the resolution of the image however, there could be an offset up to $\sim 5$ au which would result in an apocenter glow of $\sim 6.7 \%$ according to the calculation proposed in Pan et al. (2016). 

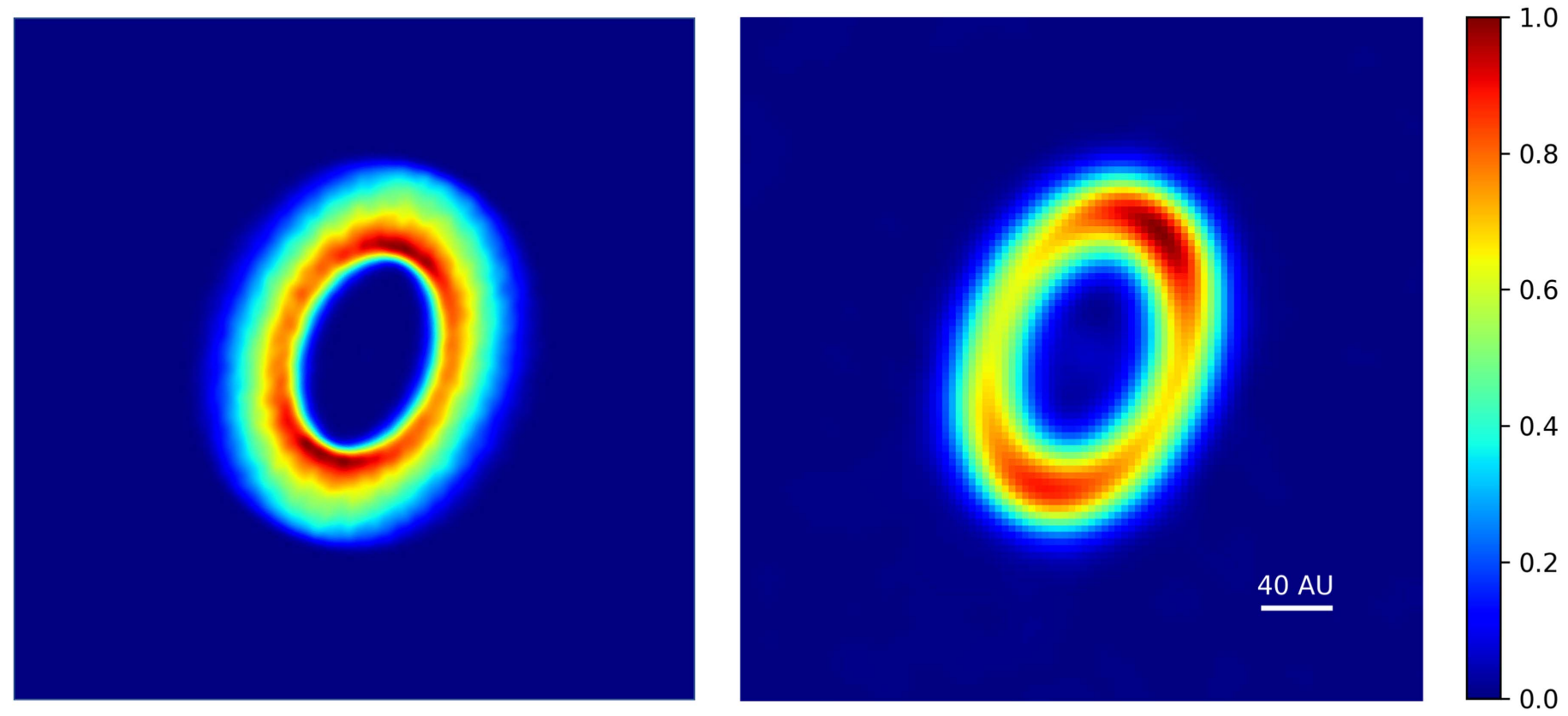

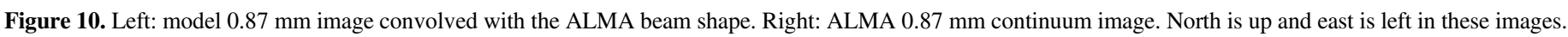
Both of the images are normalized to the brightest pixel in the image

\subsection{Differences in Gas and Dust Imagery}

Most of the gas features seen in the $\mathrm{HCO}^{+} J=4-3$ and $\mathrm{CO}$ $J=3-2$ maps do not have an analog in the $0.87 \mathrm{~mm}$ continuum or $H$-band imagery. The only notable exceptions are the excess flux in the northwest of the continuum image, an inner gap, and an inner disk, which is present in all of the available data.

\subsubsection{Azimuthal Gap in $\mathrm{HCO}^{+} \mathrm{J}=4-3$}

While some of these features are easily explained by known properties, such as the diminished, central region in the $\mathrm{CO}$ $J=3-2$ emission and the larger radial extent of both $\mathrm{HCO}^{+}$ $J=4-3$ and CO $J=3-2$ emission, of the gas the lack of an azimuthal gap in the continuum or $H$-band data is not. It is likely not a gravitational depletion of the disk by a compact body as there should be no difference in how the dust and gas are affected gravitationally. This suggests a more exotic cause for the apparent difference.

Options we have considered are a large stellar flare from a coronal mass ejection, an embedded planet, or shadowing from an inner optically thick blob of material. A large stellar flare could disturb the gas in a localized region of the disk and possibly move the dust in that region as well. This could neatly link the azimuthal gap seen in the $\mathrm{HCO}^{+} J=4-3$ data as well as the large blob of mass seen in the $0.87 \mathrm{~mm}$ continuum. A similar effect was seen in au Mic, where it is suggested that a stellar flare caused fast moving features in the dust of the disk (Boccaletti et al. 2015). However, though this could cause feature propagation in the dust disk, we would expect the density wave to travel around the disk or perhaps cause dust blowout from the impact. The difference in density from either scenario is not seen in the $0.87 \mathrm{~mm}$ continuum and there seems to be no difference in the location of the bright blob in the $1.3 \mathrm{~mm}$ continuum (Hashimoto et al. 2015) and the $0.87 \mathrm{~mm}$ continuum.

An embedded planet could cause the local region around it to undergo various chemical changes due to heating from the planet
(Cleeves et al. 2015). Though the heating would initially cause any frozen out gases to sublimate, it may also catalyze complicated chemical interactions which could locally deplete the HCO resulting in an apparent gap in the gas disk. It has been shown that a Jupiter-sized planet can cause an effect in a radius of several au surrounding the planet (Cleeves et al. 2015), which grows as the planet moves further from the star. Unfortunately a planet of this size would not be visible in the currently available data.

Shadowing from an inner disk would result in an apparent gap in the disk dust (Long et al. 2017; Stolker et al. 2016) and should similarly effect the gas if the inner disk is optically thick enough. In this instance, however, the shadowing body would necessarily have an asymmetric distribution as there is no equivalent azimuthal gap on the western side of the $\mathrm{HCO}^{+} J=4-3$ disk, only minor dimming. This could explain the apparent inner disk offset seen in the $\mathrm{HCO}^{+} J=4-3$ moment 0 data as it lies on the northeast side of the star closely aligned with the apparent gap. If this is the source of the apparent gap, then we would expect the shadow to move as the shadowing body moves around the star. This should happen within several months given that the shadowing body would appear to be at a radius of $\sim 5 \mathrm{au}$.

\subsubsection{Gas-poor Inner Disk Region}

As previously mentioned the SED suggests the existence of dust close to sublimation radius of the star, which is further supported by our modeling. In contrast, we see in our SpeX data (discussed more thoroughly in the Appendix) that the innermost regions of the disk, less than $1 \mathrm{au}$, seem to be devoid of gas as there is no net emission for hydrogen or $\mathrm{CO}$ gas (Figure 15) above photospheric. There is however some Ca II emission which suggests that there is chromoshperic activity similar to what is seen in HD 163296 (Sitko et al. 2008). The lack of gas emission close to the star suggests that there is also no active accretion of gas onto the star. 

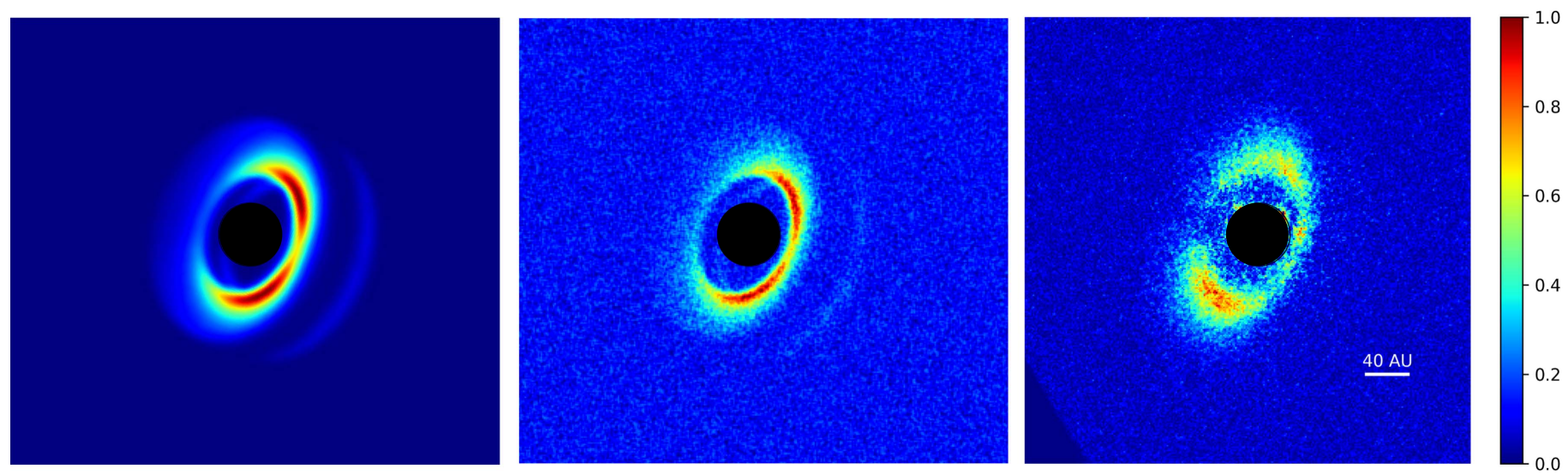

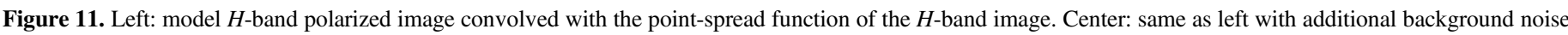

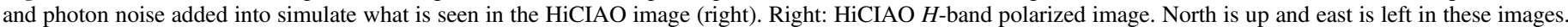

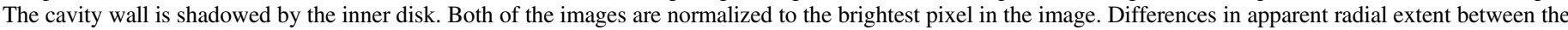
model and data are likely due to noise in the data similar to what is seen in the center image.

Table 1

The Parameters Used for the Final Model Are Shown Here

\begin{tabular}{lcl}
\hline \hline \multicolumn{2}{c}{ Model Parameters } \\
\hline Parameter & Value & Source \\
\hline Stellar Radius & $1.39 R_{\odot}$ & (Gregorio-Hetem \& Hetem 2002) \\
Stellar Mass & $0.82 M_{\odot}$ & (Riaud et al. 2006) \\
Stellar Distance & $140 \mathrm{pc}$ & (Riaud et al. 2006) \\
Stellar Temperature & $4400 \mathrm{~K}$ & (Gregorio-Hetem \& Hetem 2002) \\
Spectral Type & K5V & (Gregorio-Hetem \& Hetem 2002) \\
Small Grains & ISM & (Kim et al. 1994) \\
SG Inner Radius & $0.05 \mathrm{au}$ & $\ldots$ \\
SG Outer Radius & $110 \mathrm{au}$ & $\ldots$ \\
Large Grains & Model 2 & (Wood et al. 2002) \\
LG Inner Radius & $60 \mathrm{au}$ & $\ldots$ \\
LG Outer Radius & $110 \mathrm{au}$ & $\ldots$ \\
Disk Inclination & 45 & $\ldots$ \\
Gap & $15-60 \mathrm{au}$ & $\ldots$ \\
Disk Mass & $0.42 M_{\mathrm{J}}$ & $\ldots$ \\
\hline
\end{tabular}

Note. For the small grains we used the standard ISM dust model from Kim et al. (1994) composed of silicates and graphite, a radial power-law exponent of $\sim 3.5$, and a maximum particle size $\leqslant 0.2 \mu \mathrm{m}$. For the large grains we used model 2 from Wood et al. (2002) composed of astronomical silicates and amorphous graphite, a radial power-law exponent of $\sim 3.5$, and a maximum particle size $\leqslant 1 \mathrm{~mm}$.

\subsubsection{Bar Across $C O$}

The CO $J=3-2$ moment 1 data reveals what appears to be a bar along the minor axis of the disk. At opposite sides of the disk, directly adjacent to the bridge feature there appears to be dips in brightness in the $\mathrm{CO}$ moment 1 map. If the bridge feature is optically thick, as expected from $\mathrm{CO}$ gas, it could in turn shadow the gas in the outer disk. This however raises the question as to how the bridge structure could have formed. It could be a mass transfer between the inner and outer disk, though over time we would expect that the bridge would not remain solely along the minor axis, but instead would smear out due to Keplerian motion. We would also expect that some dust would be transferred as well, which is not seen in the continuum data. This suggests it could lie in a plane nearly perpendicular to the plane of the outer disk. However, as mentioned in Section 3, there appears to be no deviation from Keplerian motion in the vicinity of the bridge. The lack of deviation also means it is likely not an outflow from the inner disk as well.

\section{Conclusion}

In this paper we presented the image of the dust continuum at $0.87 \mathrm{~mm}$, the $\mathrm{HCO}^{+} J=4-3$ emission, and the $\mathrm{CO} J=3-2$ emission of the disks around PDS 70. We use these along with the SED of the system and $H$-band polarized data to produce an MCRT model of the system. The main results of our modeling and observation are as follows.

1. There is an azimuthal gap in the $\mathrm{HCO}^{+} J=4-3$ emission which has not been observed in other gas disks. Several possible causes include gravitational depletion by an embedded body, a large stellar flare, chemical changes due to the heating of an embedded body, and shadowing of the gas by an inner disk component. Further study is needed however to determine the most likely candidate.

2. The sub-au region of the disk is gas-poor despite evidence for a dusty inner disk component. This suggests that there is likely no gas accretion onto the star.

3. There appears to be an arm in the $\mathrm{HCO}^{+} J=4-3$ emission. The arm could be caused by a planet either very close to the star $(\sim 5 \mathrm{au})$, or in the radial gap seen in the $\mathrm{HCO}^{+} J=4-3$ and the ring seen the continuum image.

4. The CO $J=3-2$ data exhibits a bridge along it is minor axis. The bar's linear shape and the lack of deviation from Keplerian motion in the moment 1 data in that region indicate that it lies within the plane of the disk, but has not been smeared out due to Keplerian rotation. The optical thickness of $\mathrm{CO}$ gas, and the close proximity of the bridge to the two dim regions in the $\mathrm{CO} J=3-2$ emission, suggest the bridge could be shadowing the gas disk.

5. MCRT modeling, $0.87 \mathrm{~mm}$ continuum, and $H$-band polarized data suggest that PDS 70 has a two-component dust disk. The inner disk component extends from the sublimation radius out to $\sim 15$ au and is followed by a radial gap to $\sim 60 \mathrm{au}$. The outer disk component then extends from $\sim 60$ au to $\sim 110$ au.

6. We do not observe a difference between the small and large dust grain locations in the outer disk as proposed in previous work. Our $0.87 \mathrm{~mm}$ continuum image, which probes large grains on the order of $1 \mathrm{~mm}$ in size, exhibits 
a similar radial extent to that of the $H$-band polarized imagery, which probes small grains. The difference between our findings and that of Hashimoto et al. (2015) is likely due to the low angular resolution of the SMA data when compared to the ALMA data.

7. The CO $J=3-2$ moment 1 measurements suggest a dynamical mass of PDS 70 of $0.6-0.8 M_{\odot}$ similar to what has been seen in Hashimoto et al. (2015) and is consistent with the extended kinematics around a K5 star.

In this paper we have discussed various features that appear in the gas disk of PDS 70 but are not present in the dust disk. Although we cannot determine a definitive cause for these features with existing data, their existence does provide new incentives to observe this disk with other high angular resolution, high contrast instruments such as VLTI MATISSE or the NIRISS aperture masking interferometry mode of the James Webb Space Telescope. These instruments would allow us to probe the inner regions of the disk where we suspect that large planets could be causing many of these features.

We also discussed the overall structure of the dust disk found from the continuum data and MCRT modeling. The disk seems to have no strong segregation between the dust grain sizes in the outer disk, but the inner disk does appear to be devoid of large grains. This is contrary to what is suggested in previous work, but is supported by the large silicate peaks in the SED. Further investigation of the inner disk is necessary to confirm its radial extent and optical depth, and may be accomplished through use of the instruments mentioned above.

This work is supported supported by the NASA XRP grants NNX17AF88G and NNX16AJ75G. MC thanks the support from the Centro de Astrofísica de Valparaíso. S.K. acknowledges support from an STFC Rutherford Fellowship (ST/J004030/1) and ERC Starting Grant (Grant Agreement No. 639889). This work is supported by the Astrobiology Center Program of National Institutes of Natural Sciences (NINS) (Grant Number: AB281013) and by MEXT KAKENHI No. 17K05399 (EA). Y.H. is currently supported by Jet Propulsion Laboratory, California Institute of Technology, under a contract from NASA. This paper makes use of the following ALMA data: ADS/JAO.ALMA\#2015.1.00888. S. ALMA is a partnership of ESO (representing its member states), NSF (USA) and NINS (Japan), together with NRC (Canada), MOST and ASIAA (Taiwan), and KASI (Republic of Korea), in cooperation with the Republic of Chile. The Joint ALMA Observatory is operated by ESO, AUI/NRAO, and NAOJ. The National Radio Astronomy Observatory is a facility of the National Science Foundation operated under cooperative agreement by Associated Universities, Inc.

\section{Appendix}

\section{A.1. SpeX and REM Observations of PDS 70}

PDS 70 was observed on 2017 January 13 UT using the SpeX spectrograph (Rayner et al. 2003) on NASA's Infrared Telescope Facility (IRTF). For these observations the SXD grating $(0.7-2.4 \mu \mathrm{m})$ and 0.8 arcsec wide slit were used. Data reduction was done using the Spextool package (Cushing et al. 2004) running under IDL. Telluric correction and flux calibration (Vacca et al. 2003) were done using the A0V star HD 130163.

We also observed PDS 70 on 2016 June 10 UT using the Rapid Eye Mount (REM) telescope in the $g^{\prime} r^{\prime} i^{\prime} z^{\prime} J H$ filters. Two

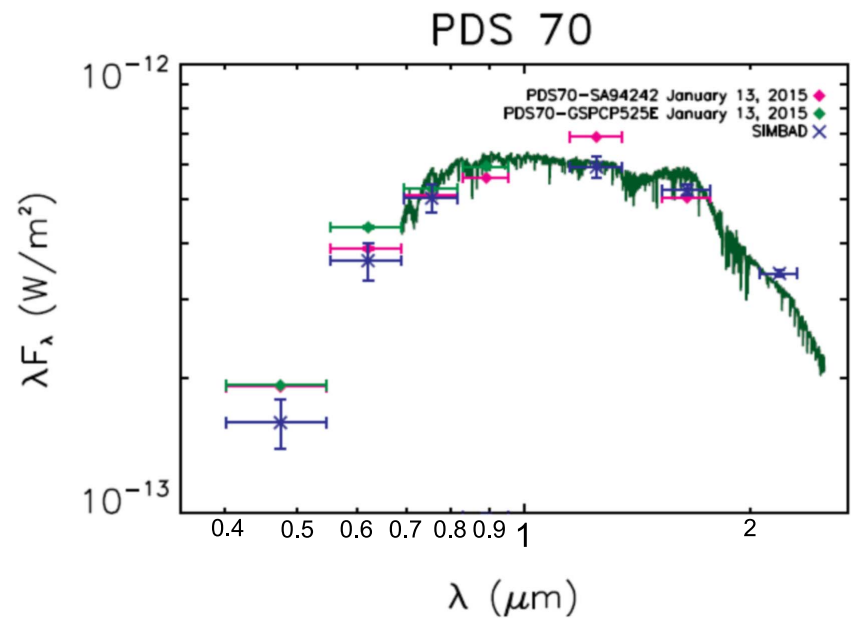

Figure 12. REM Data in the $g^{\prime} r^{\prime} i^{\prime} z^{\prime} J H$ filters. The green diamonds are data using the flux calibration star GSPC-P525E and the pink diamonds are data using the flux calibration star SA94-242. The blue Xs are both the $\mathrm{g}^{\prime} \mathrm{r}^{\prime} \mathrm{i}^{\prime}$ data from the AAVSO Photometric All Sky Survey and the JHK data from the 2MASS survey. The green line is the SpeX data for PDS 70 .

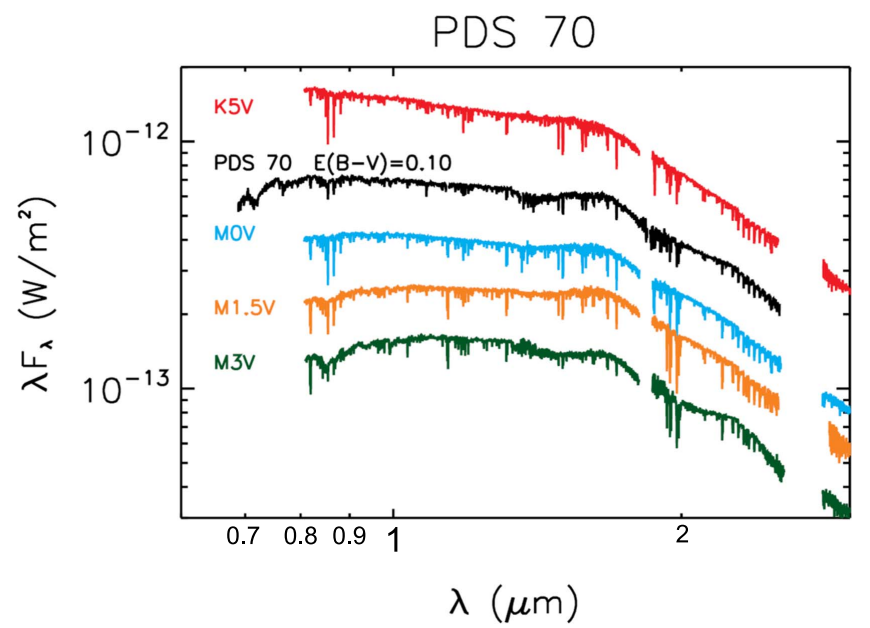

Figure 13. Our SpeX spectrum data in black (dereddened by $E(B-V)=0.10$ ) with other spectrum data gathered from the IRTF Spectral Library for K5V, $\mathrm{M} 0 \mathrm{~V}, \mathrm{M} 1.5 \mathrm{~V}$, and $\mathrm{M} 3 \mathrm{~V}$ stars.

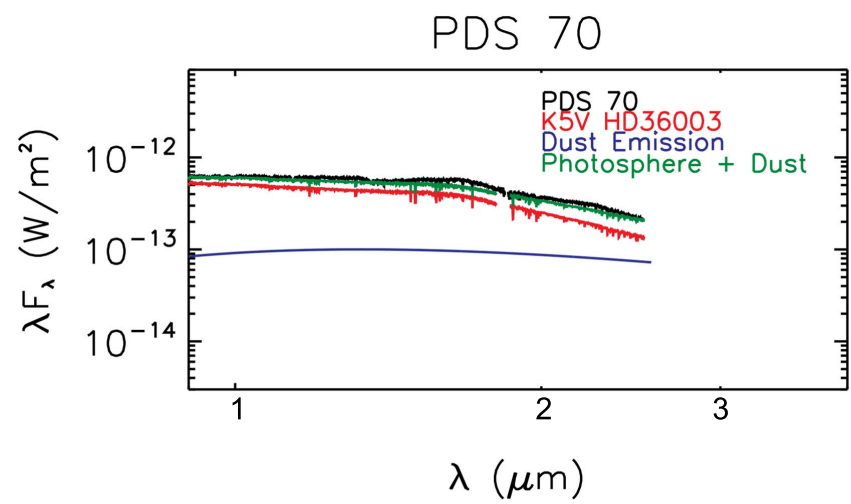

Figure 14. Model for PDS 70. The SED consists of the photospheric emission of the star plus the thermal emission by the inner dust. The relative strengths of these two components were adjusted to match the observed emission of the star plus the disk system. The process used is fully described in Sitko et al. (2012).

flux calibration stars were used, SA94-242 and GSPC-P525E. Our REM data is shown in Figure 12, along with the $g^{\prime} r^{\prime} i^{\prime}$ data from the AAVSO Photometric All Sky Survey (APASS; 

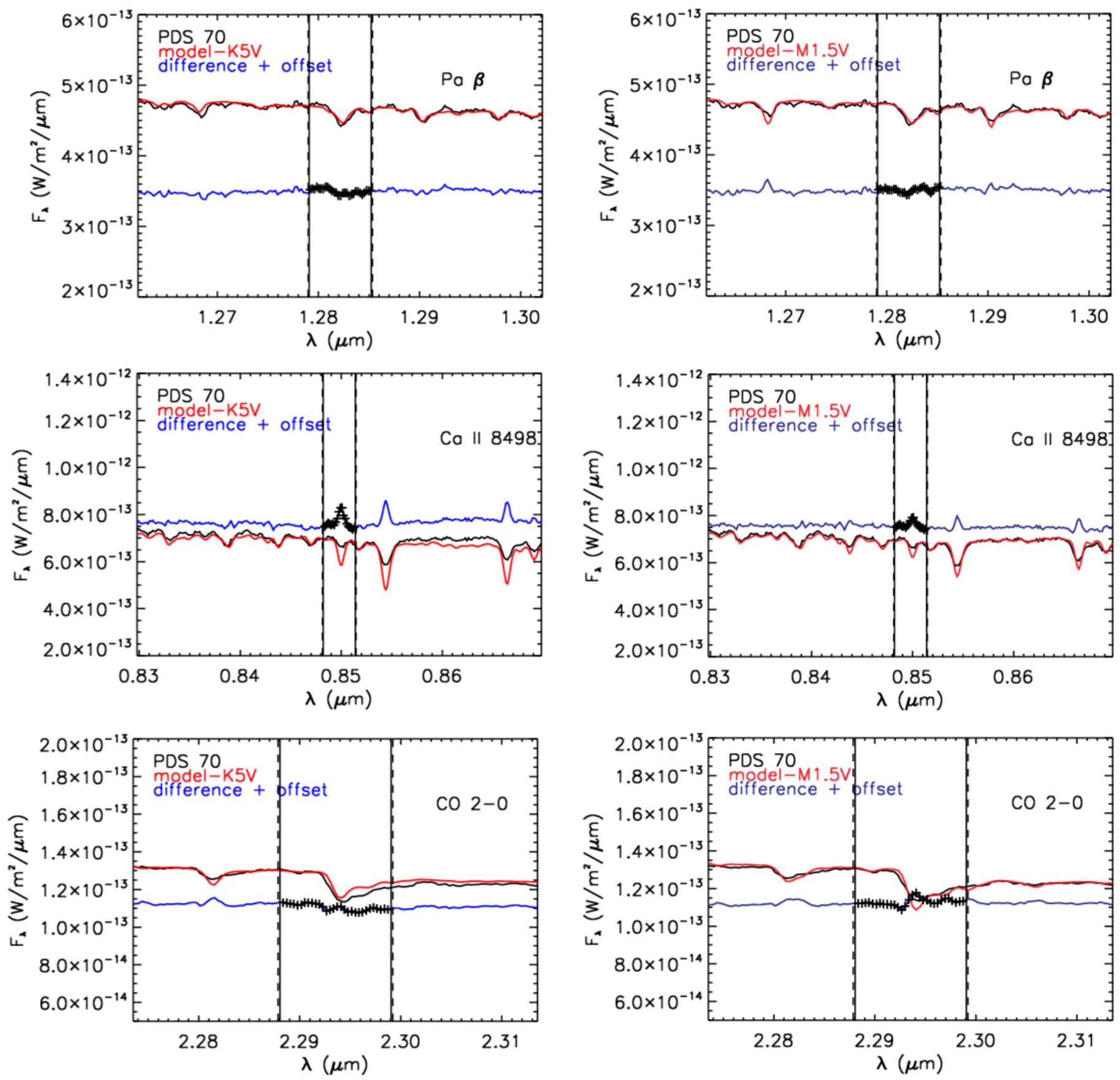

Figure 15. Line extractions from SpeX for $\mathrm{Pa} \beta, \mathrm{CO}(2-0)$, and $\mathrm{Ca}$ II 8498 for PDS 70. The red line is our best-fit model for the emission, and the blue line is the difference between the two plus an offset. We performed line extractions for both K5V (left) and M1.5V (right) model stars.

Henden et al. 2016) and JHK data from the 2MASS survey (Skrutskie et al. 2006). These were used to determine the approximate absolute fluxes for the SXD data.

In Figure 13 we compare our SpeX spectrum with a sample of similar data sets from the IRTF Spectral Library (http://irtfweb. ifa.hawaii.edu/ spex/IRTF_Spectral_Library/). Despite K5V classification usually adopted for the star (Gregorio-Hetem \& Hetem 2002; Riaud et al. 2006), at near-IR wavelengths it resembles an early $M$ star. Similar differences in classification between visible and IR wavelengths have been seen before in other stars such as TW Hydrae (Debes et al. 2013) and V4046 Sgr (Kastner et al. 2014).

\section{A.2. Gas Accretion in the PDS 70 System}

To determine the potential gas accretion characteristics in PDS 70, we subtracted off a spectral model consisting of the photospheric flux and the thermal contribution of the dust (Figure 14), in a manner similar to that of Sitko et al. (2012). For the template photosphere, we used the SpeX spectrum of a K5V star. Despite the fact that Gregorio-Hetem \& Hetem (2002) found $\mathrm{H} \alpha$ emission, we find that the $\mathrm{Pa} \beta$ line commonly used as a measure of accretion in PMS stars showed no detectable emission (Figure 15). The CO bandhead emission between 2.3 and $2.4 \mu \mathrm{m}$ that are sometimes seen in emission in gas-rich debris disks also look 


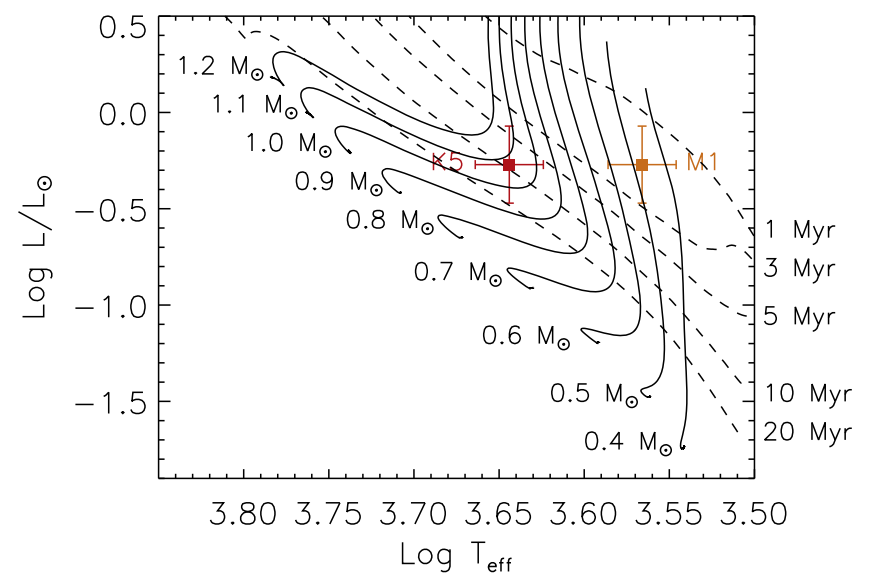

Figure 16. Location of PDS 70 relative to the PMS evolutionary tracks and isochrones of Tognelli et al. (2011). The two locations of PDS 70 are derived using an assumed spectral type of K5 (red point) and M1 (orange point), and produce different mass and age estimates.

as if there is no appreciable $\mathrm{CO}$ in emission. In contrast, the lines of Ca II are weakly in emission. It is known that in late type PMS stars, lines of Ca II can be in emission when accretion is otherwise weak or absent (Ingleby et al. 2011), suggesting that this line, and possibly $\mathrm{H} \alpha$, are chomospheric in origin, and not due to gas accretion onto the star. These data suggest that the inner regions of the disk system in PDS 70 are gas-poor, and that the $\mathrm{CO}$ gas detected with ALMA in the inner regions of PDS 70 do not extend down to the star itself. This is also suggested from CO $J=3-2$ moment 1 data where we can infer that the gas only extends down to $\sim 5 \mathrm{au}$.

\section{A.3. Mass and Age of PDS 70}

Using the V magnitude of PDS 70 (Kiraga 2012), the 140 pc distance from the association with the Sco-Cen Association, and the bolometric correction and initially adopting an effective temperature for a K5 PMS stars from Pecaut \& Mamajek (2013) we derived a luminosity of $0.65 L_{\odot}$. Figure 16 shows the location of PDS 70 relative to the PMS evolution tracks and isochrones (Tognelli et al. 2011), for $z=0.02$, helium mass fraction of 0.27 , convective parameter $\alpha=1.68$, and a deuterium abundance of $2 e^{-5}$. The online versions of these use the abundances of Asplund et al. (2005) but are nearly indistinguishable from those based on the revised abundances of Asplund et al. (2009), as seen in the figures in Tognelli et al. (2011). From these we derived a mass of $1.1 M_{\odot}$ and an age of 10 Myr. If instead, we do the same determination for an M1V classification, the mass drops to $0.5 M_{\odot}$ and the age to around 2 Myr. Based on the equivalent width of the Li lines in PDS 70 from Gregorio-Hetem \& Hetem (2002), Metchev et al. (2004) derive an age of $\leqslant 10 \mathrm{Myr}$. The location in the sky would put PDS 70 in the Upper Centaurus Lupus (UCL) region (Preibisch \& Mamajek 2008) of the Sco-Cen Association. Song et al. (2012) have looked at a large number of G, K, and M stars in the Sco-Cen complex. Based on PMS evolutionary tracks and Li line strengths, they find an age of $\sim 10 \mathrm{Myr}$ for this region.
This number is consistent with the age we obtained for the $\mathrm{K} 5 \mathrm{~V}$ classification for PDS 70.

\section{ORCID iDs}

Zachary C. Long (D) https://orcid.org/0000-0002-2048-5741 Eiji Akiyama (iD https://orcid.org/0000-0002-5082-8880 Michael Sitko (10 https://orcid.org/0000-0003-1799-1755 Ruobing Dong (i) https://orcid.org/0000-0001-9290-7846 Misato Fukagawa (iD https://orcid.org/0000-0003-3500-2455 Shu-Ichiro Inutsuka (i) https://orcid.org/0000-0003-4366-6518 Stefan Kraus (1) https://orcid.org/0000-0001-6017-8773 Jungmi Kwon (iD https://orcid.org/0000-0003-2815-7774 Carey M. Lisse (iD https://orcid.org/0000-0002-9548-1526 Michihiro Takami (i) https://orcid.org/0000-0001-9248-7546 Motohide Tamura (iD https://orcid.org/0000-0002-6510-0681 Thayne Currie (i) https://orcid.org/0000-0002-7405-3119 John P. Wisniewski (iD https://orcid.org/0000-0001-9209-1808

\section{References}

Alves, F. O., Girart, J. M., Caselli, P., et al. 2017, A\&A, 603, L3 Asplund, M., Grevesse, N., \& Sauval, A. J. 2005, in ASP Conf. Ser. 336, Cosmic Abundances as Records of Stellar Evolution and Nucleosynthesis, ed. T. G. Barnes, III \& F. N. Bash (San Francisco, CA: ASP), 25

Asplund, M., Grevesse, N., Sauval, A. J., \& Scott, P. 2009, ARA\&A, 47, 481 Boccaletti, A., Thalmann, C., Lagrange, A.-M., et al. 2015, Natur, 526, 230 Casassus, S., van der Plas, G., Sebastian Perez, M., et al. 2013, Natur, 493, 191 Cleeves, L. I., Bergin, E. A., \& Harries, T. J. 2015, ApJ, 807, 2 Cushing, M. C., Vacca, W. D., \& Rayner, J. T. 2004, PASP, 116, 362

Debes, J. H., Jang-Condell, H., Weinberger, A. J., Roberge, A., \& Schneider, G. 2013, ApJ, 771, 45

Dong, R., Hashimoto, J., Rafikov, R., et al. 2012, ApJ, 760, 111

Dong, R., Zhu, Z., Rafikov, R. R., \& Stone, J. M. 2015, ApJL, 809, L5

Edgar, R. G., \& Quillen, A. C. 2008, MNRAS, 387, 387

Gregorio-Hetem, J., \& Hetem, A. 2002, MNRAS, 336, 197

Hashimoto, J., Dong, R., Kudo, T., et al. 2012, ApJL, 758, L19

Hashimoto, J., Tsukagoshi, T., Brown, J. M., et al. 2015, ApJ, 799, 43

Henden, A. A., Templeton, M., Terrell, D., et al. 2016, yCat, 2336

Ingleby, L., Calvet, N., Bergin, E., et al. 2011, ApJ, 743, 105

Kastner, J. H., Hily-Blant, P., Rodriguez, D. R., Punzi, K., \& Forveille, T. 2014, ApJ, 793, 55

Kim, S.-H., Martin, P. G., \& Hendry, P. D. 1994, ApJ, 422, 164

Kiraga, M. 2012, yCat, 1206

Kraus, S., Kreplin, A., Fukugawa, M., et al. 2017, ApJL, 848, L11 Long, Z. C., Fernandes, R. B., Sitko, M., et al. 2017, ApJ, 838, 62

Matter, A., Labadie, L., Augereau, J. C., et al. 2016, A\&A, 586, A11 Metchev, S. A., Hillenbrand, L. A., \& Meyer, M. R. 2004, ApJ, 600, 435 Öberg, K. I., Qi, C., Fogel, J. K. J., et al. 2010, ApJ, 720, 480 Pan, M., Nesvold, E. R., \& Kuchner, M. J. 2016, ApJ, 832, 81 Pecaut, M. J., \& Mamajek, E. E. 2013, ApJS, 208, 9

Preibisch, T., \& Mamajek, E. 2008, in Handbook of Star Forming Regions, Vol. II, ed. B. Reipurth (San Francisco, CA: ASP), 235

Rayner, J. T., Toomey, D. W., Onaka, P. M., et al. 2003, PASP, 115, 362 Riaud, P., Mawet, D., Absil, O., et al. 2006, A\&A, 458, 317

Sitko, M. L., Carpenter, W. J., Kimes, R. L., et al. 2008, ApJ, 678, 1070

Sitko, M. L., Day, A. N., Kimes, R. L., et al. 2012, ApJ, 745, 29

Skrutskie, M. F., Cutri, R. M., Stiening, R., et al. 2006, AJ, 131, 1163

Song, I., Zuckerman, B., \& Bessell, M. S. 2012, AJ, 144, 8

Stolker, T., Dominik, C., Avenhaus, H., et al. 2016, A\&A, 595, A113

Tognelli, E., Prada Moroni, P. G., \& Degl'Innocenti, S. 2011, A\&A, 533, A109 Vacca, W. D., Cushing, M. C., \& Rayner, J. T. 2003, PASP, 115, 389

Whitney, B. A., Robitaille, T. P., Bjorkman, J. E., et al. 2013, ApJS, 207, 30 Whitney, B. A., Wood, K., Bjorkman, J. E., \& Wolff, M. J. 2003, ApJ, 591, 1049

Wood, K., Wolff, M. J., Bjorkman, J. E., \& Whitney, B. 2002, ApJ, 564, 887 